\title{
Entropic Stabilization of Water at Graphitic Interfaces
}

Tod A Pascal ${ }^{1,2 *}$ and William A. Goddard III $^{3}$

${ }^{1}$ ATLAS Materials Physics Laboratory, Department of NanoEngineering and Chemical Engineering, University of California San Diego, La Jolla, CA 92093

${ }^{2}$ Materials Science and Engineering, University of California San Diego, La Jolla, CA 92093

${ }^{3}$ Materials and Process Simulation Center, California Institute of Technology, Pasadena, CA 91125

Corresponding author: tpascal@ucsd.edu (T.A.P.)

\section{Supporting Information}

\section{Table of Contents}

1. Computational Methods............................................................................................................. 2

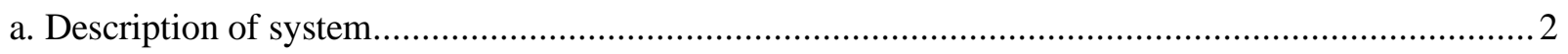

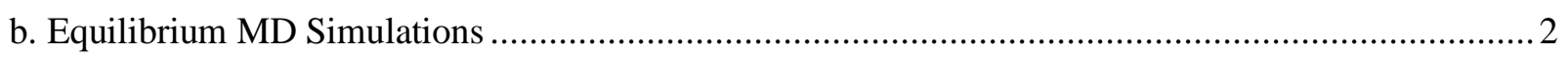

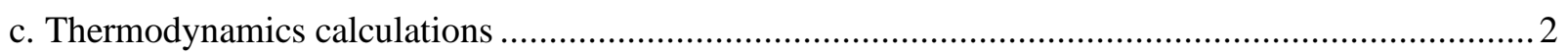

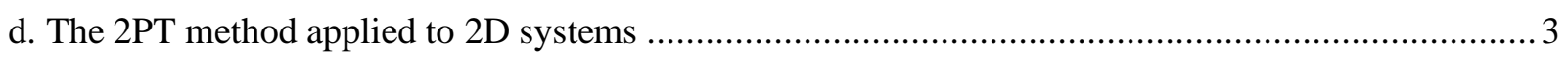

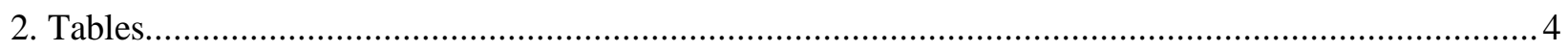

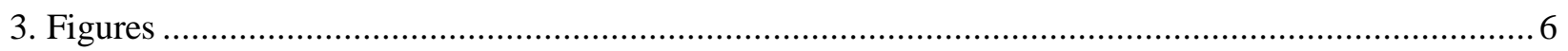

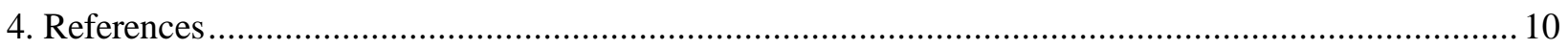




\section{Computational Methods}

\section{a. Description of system}

We created supercells of graphene sheets by replicating the fundamental unit cell by $9 \times 16$, to a $2 \mathrm{D}$ periodic sheet of 38.4 x $39.5 \AA^{2}$ (586 atoms). Multi-sheet structures were created assuming a $3.33 \AA$ sheetsheet separation. Composite systems were created by encapsulating a box of 4353 water molecules, described with the rigid TIP4P-2005 forcefield ${ }^{1}$, pre-equilibrated from 10ns constant pressure (1bar), constant temperature (298K) MD simulations using LAMMPS MD simulation engine ${ }^{2}$. In these preequilibration simulations, the $\mathrm{x}$ and $\mathrm{y}$ dimensions were fixed to be commensurate to the graphene sheet dimensions, while the $\mathrm{z}$-dimension was adjusted to achieve a $1 \mathrm{~g} / \mathrm{cm} 3$ density, resulting in a final $\mathrm{z}$ dimension of $93.06 \AA$. We allowed for a $2.0 \AA$ buffer between the topmost graphene sheet and the water surface. Finally, we inflated the box in the z-direction by $2 \mathrm{~nm}$, in order to simulate a vacuum on either side of our graphene sheets.

\section{b. Equilibrium MD Simulations}

We subjected each of the initial systems to long-term MD simulations with an additional force of \pm 0.022 $\mathrm{kcal} / \mathrm{mol} / \AA$ added to the top and bottom sheets respectively, in order to simulate 1atm of external pressure. The graphene sheets were described with the QMFF-Cx forcefield ${ }^{3}$. The water-carbon van der Waals interactions were determined using the optimized parameter set from Werder et $\mathrm{al}^{4}$. We then performed 2D periodic ( $\mathrm{x}$ and $\mathrm{y}$ dimension) equilibrium MD simulations using LAMMPS. The top and bottom of the simulation box were bounded by a purely repulsive wall. In order to remove spurious interactions between the two surfaces, we employed the 2D slab corrections of Yeh and Berkowitz ${ }^{5}$ with a further $3.0 \mathrm{z}$-factor.

We initially minimized the forces in each system using the conjugate gradient minimization scheme with an energy tolerance of $10^{-4}$ and a force tolerance of $10^{-5}$. We then slowly heated the system from $0 \mathrm{~K}$ to $298 \mathrm{~K}$ over $100 \mathrm{ps}$ in the constant volume, constant temperature NVT (canonical) ensemble, using a Nose-Hoover thermostat (temperature relaxation constant of $100 \mathrm{fs}$ ). The equations of motion were integrated by means of the velocity verlet algorithm in a $1 \mathrm{fs}$ timestep. We truncated the van der Waals forces at an outer cutoff of $1.2 \mathrm{~nm}$ and an inner cutoff of $1.1 \mathrm{~nm}$, between which we employed a cubic spline to guarantee zero energy and forces at the outer cutoff. We truncated the short-range electrostatic interactions at $1.2 \mathrm{~nm}$ and evaluated the long-range electrostatics using the 2D-PPPM method ${ }^{6}$ with a kspace tolerance of $10^{-6} \mathrm{kcal} / \mathrm{mol}$.

After initial equilibration, we annealed the system by cycling the temperature 5 times between $298 \mathrm{~K}$ and $550 \mathrm{~K}$ over $30 \mathrm{ps}$, followed by a further $100 \mathrm{ps}$ of NVT dynamics at $298 \mathrm{~K}$. We resolved any x and y stresses in the system by means of $1 \mathrm{~ns}$ of constant pressure, constant temperature (NPT) simulations using the Andersen barostat (pressure relaxation constant of $1 \mathrm{ps}$ ). The equations of motion used are those of Shinoda et al. ${ }^{7}$, which combine the hydrostatic equations of Martyna et al. ${ }^{8}$ with the strain energy proposed by Parrinello and Rahman ${ }^{9}$. The time integration schemes closely follow the time-reversible measure-preserving Verlet integrators derived by Tuckerman et al. ${ }^{10}$. During the last 500 ps of the $1 \mathrm{~ns}$ NPT simulation, we calculated the average $\mathrm{x}$ and $\mathrm{y}$ cell lengths and adjusted the final NPT simulation cell linearly to the averages over a further 100 ps of dynamics. Finally, we performed 30 cycles of NVT dynamics, where the temperature was cycled from $298 \mathrm{~K}$ to $550 \mathrm{~K}$ over $250 \mathrm{ps}$, followed by $250 \mathrm{ps}$ of NVT simulation at $298 \mathrm{~K}$. We saved snapshots of the system (atomic position and velocities) every $10 \mathrm{ps.}$

\section{c. Thermodynamics calculations}

We saved a restart file after each cycle and used a starting point for an additional $25 \mathrm{ps}$ of NVT dynamics, saving the atomic positions and velocities every $2 \mathrm{fs}$. We then calculated the system thermodynamics 
using an external code that implements the 2PT method. In each 2PT calculation, we took the effective volume of the nanoconfined water molecules to be an enclosed volume with $z=D-3.75 \AA$, which accounts for the carbon-water van der Waals exclusion zone. We obtained the mean and standard deviation in our calculated thermodynamics and DoS functions from statistical averaging over the 2PT results of all 30 snapshots for each system. We also performed reference simulations of the bulk liquids (Table S2) and the thermodynamics evaluated using the 2PT method.

\section{d. The 2PT method applied to 2D systems}

Details of the 2PT method has been presented elsewhere ${ }^{11-14}$, so we present an overview here, pointing out salient features for assessing the thermodynamics of 2D systems, and direct the interested reader to the published works ${ }^{12,15}$. In 2PT, the system thermodynamics are calculated from the density of states $\operatorname{DoS}(\mathrm{v})$ (spectral density or power spectrum), obtained by the Fourier Transform of the velocity autocorrelation function $\mathrm{C}(\mathrm{t})^{16}$ :

$$
\begin{aligned}
& C(t)=\sum_{j=1}^{N} \sum_{k=1}^{3} m_{j}\left[\lim _{t \rightarrow \infty} \frac{1}{2 \tau} \int_{-\tau}^{\tau} v_{j}^{k}\left(t^{\prime}+t\right) v_{j}^{k}\left(t^{\prime}\right) d t^{\prime}\right] \\
& \operatorname{DoS}(v)=\lim _{t \rightarrow \infty} \frac{1}{2 k T} \int_{-\tau}^{\tau} C(t) e^{-2 \pi v t} d t
\end{aligned}
$$

The total $\operatorname{DoS}(\mathrm{v})$ is then partitioned into a contribution arising from pure diffusion in the liquid $\left[D o S_{\text {diffuse }}(v)\right]$ and a contribution arising from solid like vibrations $\left[D o S_{\text {solid }}(v)\right]$, as proposed by Eyring and $\operatorname{Ree}^{17}$ :

$$
\operatorname{DoS}(v)=f * \operatorname{DoS}_{\text {diffuse }}(v)+(1-f) * \operatorname{DoS}_{\text {solid }}(v)
$$

where $\mathrm{f}$ is the "fluidicity factor": the fraction of the modes of the system that are diffusional. This f factor is function of the system properties (self-diffusion, density and temperature) and is solved self consistently from the MD trajectory. In the original formulation, the total system thermodynamics is then recovered by integrating the individual power spectrum with the appropriate weighting factors, obtained from the Carnahan-Sterling (CS) equation of state (EOS) of hard-spheres ${ }^{18}$ in the case of $D o S_{\text {diffuse }}(v)$ and from Debye theory of a vibrating crystal ${ }^{19}$ in the case of $D o S_{\text {solid }}(v)$. Recently, Maiti and coworkers showed that the power spectrum of a 2D fluid may display unphysical physics, and that converged thermodynamics of a $2 \mathrm{D}$ system is obtained by considering the thermodynamics of hard disks ${ }^{15}$. This correction is employed in the present work. Specifically, due to the large anisotropy in in-plane and outof-plane self-diffusion constant, we evaluated the autocorrelation functions in each of the $\mathrm{x}, \mathrm{y}$ and $\mathrm{z}$ directions separately. The system thermodynamics is then taken as the sum of all three contributions. This eorrection is employed in the present work. Another approach for treating the diffusive component, which is applicable to 2D systems, is the memory function approach of Desjarlais ${ }^{20}$, which we found gives similar results to that of Maiti et al. 


\section{TABLES}

Table S1: Comparison of the $298 \mathrm{~K}$ solid-liquid surface energies $\left(\mathrm{mJ} / \mathrm{m}^{2}\right)$ of water on graphene and graphite for several different water models. The total surface free energy $\left(\gamma_{\mathrm{SL}}\right)$ is separated into its enthalpic $\left(\gamma_{\mathrm{SL}}{ }^{\mathrm{H}}\right)$ and entropic $\left(\mathrm{T} \gamma_{\mathrm{SL}}{ }^{\mathrm{S}}\right.$ ) components. The surface energy difference $\gamma_{\mathrm{SL}}{ }^{{ }^{\mathbf{G}}}=\gamma_{\mathrm{SL}}{ }^{\mathbf{G}}$ (graphene) $\gamma_{\mathrm{SL}}{ }^{\mathbf{G}}$ (graphite) is provided as reference, as is the liquid-vapor surface free energy $\gamma_{\mathrm{LV}}$.

\begin{tabular}{l|ll|ll|ll|ll|ll|ll|l|l}
\hline Water FF & \multicolumn{5}{|c|}{ Graphene } & \multicolumn{5}{c|}{ Graphite } & \\
\hline & $\gamma_{\mathrm{SL}}{ }^{\mathbf{G}}$ & \pm & $\mathrm{T} \gamma_{\mathrm{SL}}{ }^{\mathrm{S}}$ & \pm & $\gamma_{\mathrm{SL}}{ }^{\mathrm{H}}$ & \pm & $\gamma_{\mathrm{SL}}{ }^{\mathbf{G}}$ & \pm & $\mathrm{T} \gamma_{\mathrm{SL}}{ }^{\mathrm{S}}$ & \pm & $\gamma_{\mathrm{SL}}{ }^{\mathrm{H}}$ & \pm & $\gamma_{\mathrm{SL}}{ }^{\mathbf{G}}$ & $\gamma_{\mathrm{LV}}$ \\
\hline $\mathbf{S P C}^{\mathbf{2 1}}{ }^{21}$ & -30.6 & 2.3 & -7.5 & 2.2 & -38.1 & 0.9 & -46.5 & 3.6 & 10.0 & 3.6 & -36.4 & 1.9 & 15.9 & 55.8 \\
\hline $\mathbf{T I P 3 P}^{\mathbf{2 2}}$ & -18.9 & 2 & -18.3 & 2.1 & -37.2 & 1.2 & -37.5 & 2.9 & 10.9 & 3.2 & -26.6 & 2.4 & -18.9 & 52.6 \\
\hline $\mathbf{T I P 4 P}^{\mathbf{2 2}}$ & -18.2 & 2.2 & 3.7 & 2.2 & -14.5 & 1.2 & -34.0 & 2.6 & 22.9 & 2.8 & -11.1 & 2.4 & -18.2 & 56.3 \\
\hline $\mathbf{T I P 4 P}^{\mathbf{2 0 0 5}}$ & -18.1 & 2.2 & 37.7 & 2.1 & 19.6 & 1.1 & -33.5 & 3.7 & 62.6 & 4.0 & 29.1 & 2.9 & -18.1 & 64.4 \\
\hline
\end{tabular}

Table S2: Comparison of the interfacial thermodynamics $\left(\mathrm{mJ} / \mathrm{m}^{2}\right)$ of water on various graphene and graphite simulated with TIP4P/2005 water model at 298K, using various water - graphene sheet interaction potentials described with a 12-6 Lennard-Jones potential $E=4 \varepsilon\left[\left(\frac{\sigma}{r}\right)^{12}-\left(\frac{\sigma}{r}\right)^{6}\right]$. We denote $\mathrm{A}_{1}=\varepsilon_{\mathrm{O}-\mathrm{C}}[\mathrm{kJ} / \mathrm{mol}], \mathrm{B}_{1}=\sigma_{\mathrm{O}-\mathrm{C}}[\AA], \mathrm{A}_{2}=\varepsilon_{\mathrm{H}-\mathrm{C}}, \mathrm{B}_{2}=\sigma_{\mathrm{H}-\mathrm{C}}$

\begin{tabular}{|c|c|c|c|c|c|c|c|c|c|c|c|c|c|c|}
\hline & & \multicolumn{6}{|c|}{ Graphene } & \multicolumn{6}{|c|}{ Graphite } & \multirow[b]{2}{*}{$\gamma_{\mathrm{SL}}{ }^{\Delta \mathrm{G}}$} \\
\hline & & $\gamma_{\mathrm{SL}}^{\mathrm{G}}$ & \pm & $\mathrm{T} \gamma_{\mathrm{SL}}{ }^{\mathrm{S}}$ & \pm & $\gamma_{\mathrm{SL}}{ }^{\mathrm{H}}$ & \pm & $\gamma_{S L}{ }^{G}$ & \pm & $\mathrm{T} \gamma_{\mathrm{SL}} \mathrm{S}^{\mathrm{s}}$ & \pm & $\gamma_{\mathrm{SL}}{ }^{\mathrm{H}}$ & \pm & \\
\hline$\underset{23}{M B-1}$ & $\begin{array}{l}\mathrm{A}_{1}=0.11 \\
\mathrm{~B}_{1}=3.32\end{array}$ & -19.5 & 1.3 & 0.0 & 4.5 & -19.5 & 0.9 & -35.2 & 2.0 & 17.0 & 6.9 & -18.2 & 1.7 & 15.7 \\
\hline$\underset{23}{\text { MB-2 }}$ & $\begin{array}{l}\mathrm{A}_{1}=0.15 \\
\mathrm{~B}_{1}=3.22\end{array}$ & -35.5 & 1.7 & -5.6 & 6.0 & -41.1 & 1.1 & -51.8 & 1.8 & 9.7 & 7.8 & -42.1 & 2.2 & 16.3 \\
\hline $\begin{array}{l}\text { QMFF- } \\
\text { cw }^{24}\end{array}$ & $\begin{array}{l}\mathrm{A}_{1}=0.11 \\
\mathrm{~B}_{1}=2.95 \\
\mathrm{~A}_{2}=0.03 \\
\mathrm{~B}_{2}=2.80\end{array}$ & -27.0 & 1.4 & -16.3 & 4.8 & -43.2 & 1.0 & -45.2 & 2.6 & 12.3 & 8.1 & -32.8 & 1.8 & 18.2 \\
\hline $\begin{array}{l}\text { Scocchi } \\
\end{array}$ & $\begin{array}{l}\mathrm{A}_{1}=0.05 \\
\mathrm{~B}_{1}=3.19\end{array}$ & 7.9 & 1.6 & 21.8 & 5.5 & 29.7 & 1.1 & -4.3 & 2.0 & 45.8 & 6.7 & 41.5 & 1.6 & 12.2 \\
\hline $\begin{array}{l}\text { Werder } \\
\end{array}$ & $\begin{array}{l}\mathrm{A}_{1}=0.09 \\
\mathrm{~B}_{1}=3.19\end{array}$ & -18.1 & 2.2 & 37.7 & 2.1 & 19.8 & 1.1 & -33.5 & 3.7 & 62.6 & 4.0 & 29.1 & 2.9 & 14.7 \\
\hline
\end{tabular}


Table S3: Decomposition of the total water energy $\left(\mathrm{H}^{\mathrm{tot}}\right)$ in water - carbon $\left(\mathrm{H}^{\mathrm{WG}}\right)$ and water - water $\left(\mathrm{H}^{\mathrm{WW}}\right)$ components $(\mathrm{N})$. Results are presented for $4353 \mathrm{SPC} / \mathrm{Ew}$ waters between electrodes comprising various numbers of graphene sheets $(\mathrm{N})$ with surface area of $3.7 \times 3.7 \mathrm{~nm}^{2}$. All energies are in $\mathrm{kJ} / \mathrm{mol}$.

\begin{tabular}{l|ll|llll}
\hline $\mathbf{N}$ & $\left\langle\mathbf{H}^{\mathbf{W W}}\right\rangle$ & $\mathbf{\pm}$ & $\left\langle\mathbf{H}^{\mathbf{W G}}\right\rangle$ & $\mathbf{\pm}$ & $\left\langle\mathbf{H}^{\mathbf{t o t}}\right\rangle$ & $\mathbf{\pm}$ \\
\hline $\mathbf{1}$ & -1273237.7 & 443.0 & -1749.4 & 34.4 & -1274987.1 & 534.0 \\
$\mathbf{2}$ & -1269528.3 & 452.6 & -1845.2 & 42.1 & -1271373.5 & 530.3 \\
$\mathbf{3}$ & -1268880.4 & 441.0 & -1903.5 & 35.5 & -1270783.9 & 530.5 \\
$\mathbf{4}$ & -1265999.6 & 427.4 & -1904.9 & 35.6 & -1267904.5 & 503.3 \\
$\mathbf{5}$ & -1262199.0 & 521.5 & -1903.8 & 36.1 & -1264102.8 & 660.2 \\
$\mathbf{6}$ & -1261659.7 & 406.7 & -1902.5 & 35.8 & -1263562.2 & 469.2 \\
\hline
\end{tabular}

Table S4: Comparison of the interfacial thermodynamics of water on multi-layer graphene sheets $(\mathrm{N})$ $298 \mathrm{~K}$ using the TIP4P/2005 water model and the graphite-water interactions of Werder et al ${ }^{4}$. All energies are in $\left[\mathrm{mJ} / \mathrm{m}^{2}\right]$.

\begin{tabular}{l|ll|ll|ll}
\hline $\mathbf{N}$ & $\boldsymbol{\gamma}_{\mathrm{SL}}$ & $\mathbf{\pm}$ & $\boldsymbol{\gamma}_{\mathrm{SL}}{ }^{\mathrm{TS}}$ & $\mathbf{\pm}$ & $\boldsymbol{\gamma}_{\mathrm{SL}}{ }^{\mathrm{H}}$ & $\mathbf{\pm}$ \\
\hline $\mathbf{1}$ & -18.1 & 2.2 & 37.7 & 2.1 & 19.6 & 1.1 \\
$\mathbf{2}$ & -29.7 & 2.5 & 47.0 & 2.5 & 17.3 & 1.4 \\
$\mathbf{3}$ & -30.9 & 2.3 & 56.0 & 2.3 & 25.1 & 1.5 \\
$\mathbf{4}$ & -32.9 & 2.7 & 62.5 & 2.8 & 29.6 & 1.8 \\
$\mathbf{5}$ & -32.2 & 3.3 & 61.6 & 3.5 & 29.4 & 2.7 \\
$\mathbf{6}$ & -33.5 & 3.7 & 62.6 & 4.0 & 29.1 & 2.9 \\
\hline
\end{tabular}

Table S5: Comparison of the interfacial thermodynamics of various water layers on graphite and graphene. All energies are in $\mathrm{mJ} / \mathrm{m}^{2}$, and distances are in $\AA$

\begin{tabular}{|l|l|l|l|l|l|l|l|l|l|l|l|l|l|}
\hline & \multicolumn{19}{|l|}{ graphene } & \multicolumn{10}{l|}{ graphite } \\
\hline & $\mathrm{z}(\AA)$ & $\left\langle\gamma_{\mathrm{sl}}{ }^{G}\right\rangle$ & \pm & $\left\langle\gamma_{\mathrm{sl}}{ }^{H}\right\rangle$ & \pm & $\left\langle\gamma_{\mathrm{sl}}{ }^{T S}\right\rangle$ & \pm & $\left\langle\gamma_{\mathrm{sl}}{ }^{G}\right\rangle$ & \pm & $\left\langle\gamma_{\mathrm{sl}}{ }^{H}\right\rangle$ & \pm & $\left\langle\gamma_{\mathrm{sl}}{ }^{T S}\right\rangle$ & \pm \\
\hline 1 & 4.81 & 4.5 & 0.3 & 18.1 & 0.3 & 13.6 & 0.4 & 2.7 & 0.4 & 21.2 & 0.3 & 18.5 & 0.3 \\
\hline 2 & 7.76 & -6.6 & 0.2 & -0.4 & 0.2 & 6.2 & 0.2 & -9.6 & 0.2 & 3.7 & 0.2 & 13.4 & 0.1 \\
\hline 3 & 11.06 & -5.8 & 0.3 & -0.3 & 0.3 & 5.5 & 0.3 & -8.5 & 0.2 & 1.8 & 0.2 & 10.3 & 0.2 \\
\hline 4 & 13.36 & -3.8 & 0.2 & -0.1 & 0.2 & 3.7 & 0.3 & -6.4 & 0.2 & 1.2 & 0.2 & 7.6 & 0.1 \\
\hline 5 & 16.66 & -3.0 & 0.2 & 0.0 & 0.2 & 2.9 & 0.3 & -4.1 & 0.2 & 0.6 & 0.2 & 4.7 & 0.2 \\
\hline 6 & 19.96 & -1.7 & 0.2 & 0.2 & 0.2 & 1.8 & 0.3 & -3.4 & 0.2 & 0.3 & 0.2 & 3.7 & 0.1 \\
\hline 7 & 23.14 & -0.7 & 0.2 & 0.0 & 0.2 & 0.7 & 0.3 & -2.0 & 0.2 & 0.1 & 0.2 & 2.1 & 0.2 \\
\hline bulk & & -1.2 & 1.1 & 2.1 & 1.0 & 3.3 & 1.1 & -2.4 & 0.9 & 0.2 & 1.0 & 2.6 & 0.9 \\
\hline
\end{tabular}




\section{FigURES}

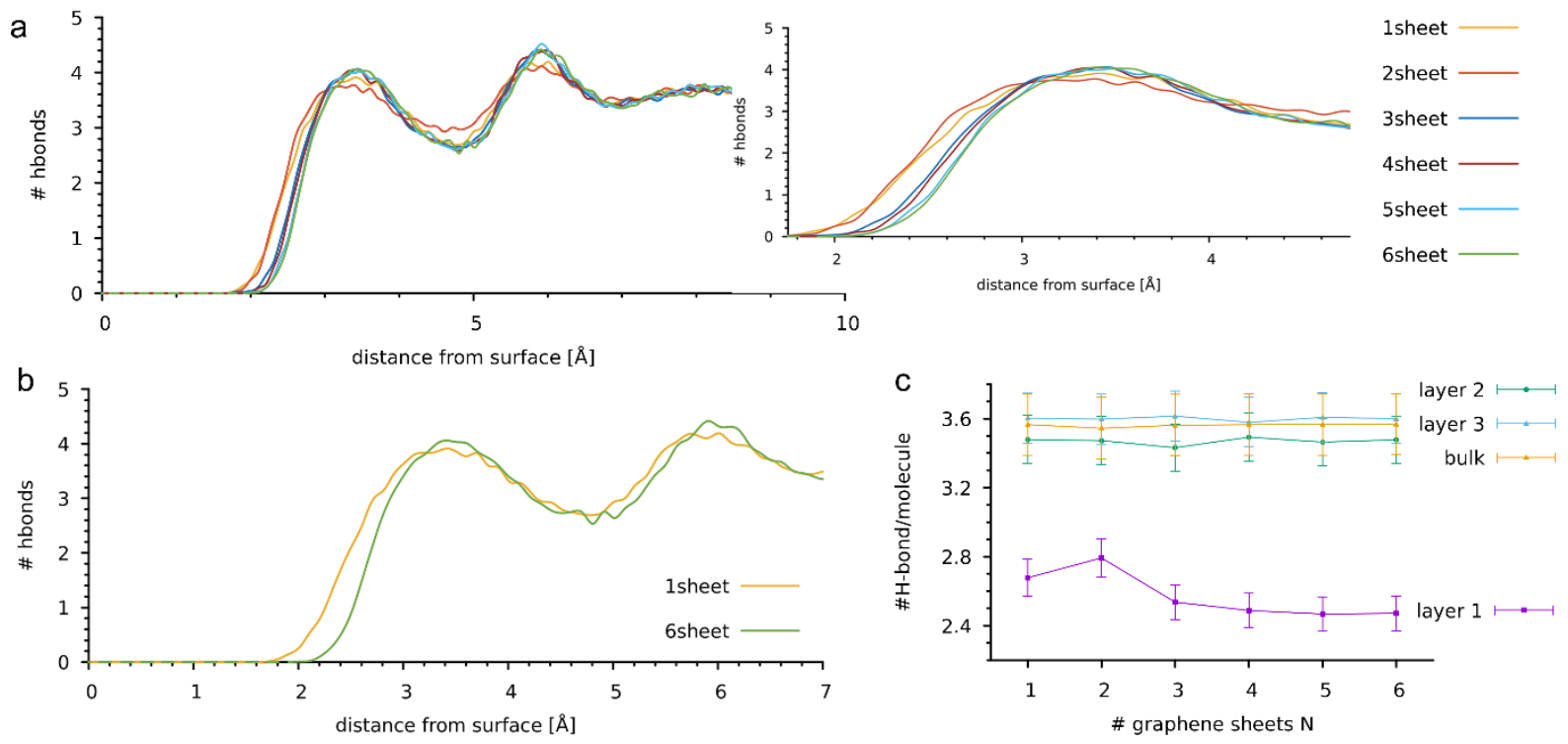

Figure S1 | Water hydrogen bonding at graphitic surfaces. a. Profile of water hydrogen bonding as a function of distance from topmost graphene surface, as a function of increasing number of graphene layers, from graphene (1sheet - orange) to graphite (6 sheets - green). We use the hydrogen bonding definition of Chandler and coworkers ${ }^{26}$. Inset: Zoom of the $1^{\text {st }}$ solvation shell H-bonding profile. $\mathbf{b}$. Comparison of the $\mathrm{H}$-bonding between graphene and graphite. 

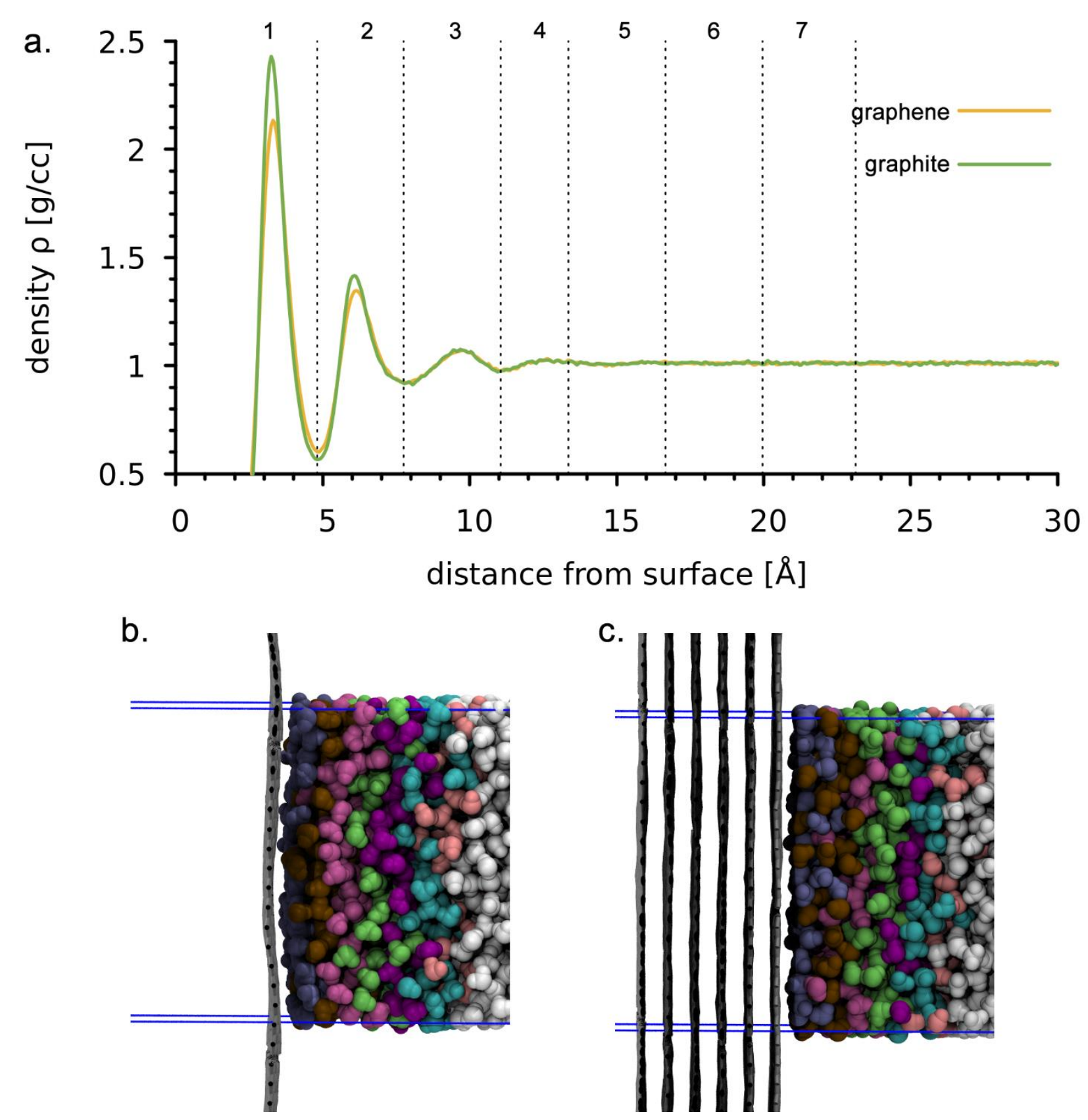

Figure S2 | Water layering on graphene and graphite. a. Mass density profile from equilibrium MD simulations. We assign the water molecule to a specific grid point ( $0.1 \AA$ grid spacing), based on the distance between the center of mass of the molecule and the minimum distance a carbon atom. The density profile is the average over all snapshots in the MD simulation. Vertical dashed lines indicate the various water shell distances, given in Table S5. b. Water layers on graphene. Snapshot is from last structure of a 20ns MD simulation. Over 40ps, we assign each water molecule to a layer as defined in a. and color the water molecules based on their average layer: layer 1 - blue, layer 2: brown, layer 3: magenta, layer 4: green, layer 5: purple, layer 6: cyan, layer 7 cyan and bulk white. c. Water layers on graphite. 

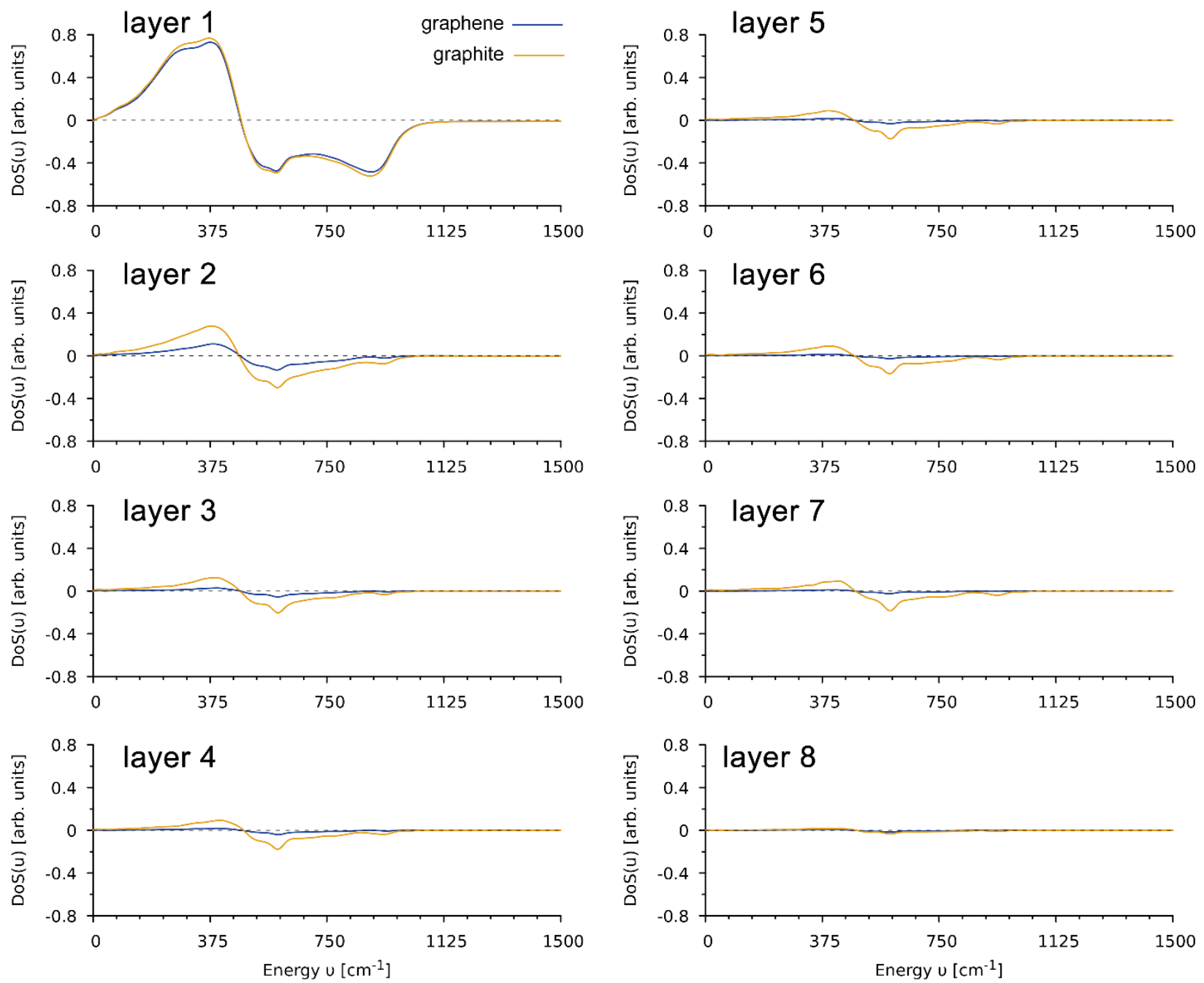

Figure S3 | Water layer Differential DoS: Comparison of the full density of states function for graphene (blue) and graphite (brown) as a function of water layer (i.e. distance from the interface). 


\section{1st water layer Internal Vibrations}

Power Spectrum

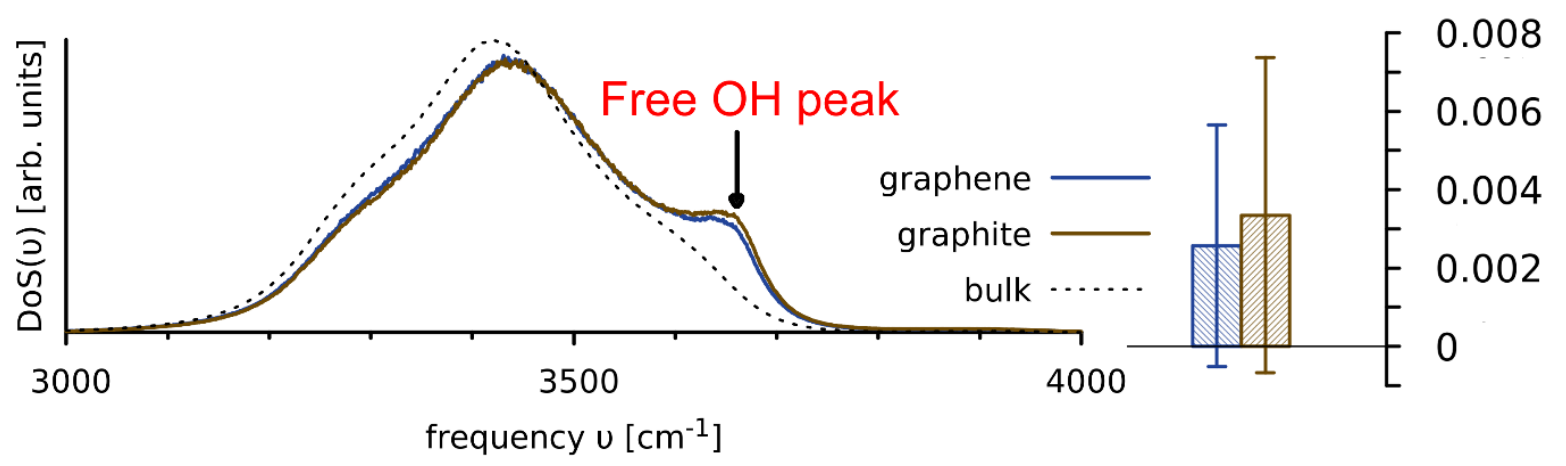

Figure S4 | Vibrational Spectra of Interfacial Water. Density of states (DoS) function of the $1^{\text {st }}$ interfacial water layer on graphene (blue) and graphite (brown) due to internal $\mathrm{OH}$ stretching interactions using the flexible TIP4P/2005f water model ${ }^{27}$. The DoS of bulk water (black dashed line) is given as a reference. Interfacial water molecules contain the signature of broken, free $\mathrm{OH}$ bonds, as an additional peak in the spectrum near $3660 \mathrm{~cm}^{-1}$ (as indicated). The spectrum is also blue shifted by $\sim 25 \mathrm{~cm}^{-1}$ compared to the bulk. The net result in an increase in the internal vibrational entropy which stabilizes the interfce (Right inset), however the high frequency of these modes means that they contribution less than $0.1 \%$ to the interfacial surface free energy

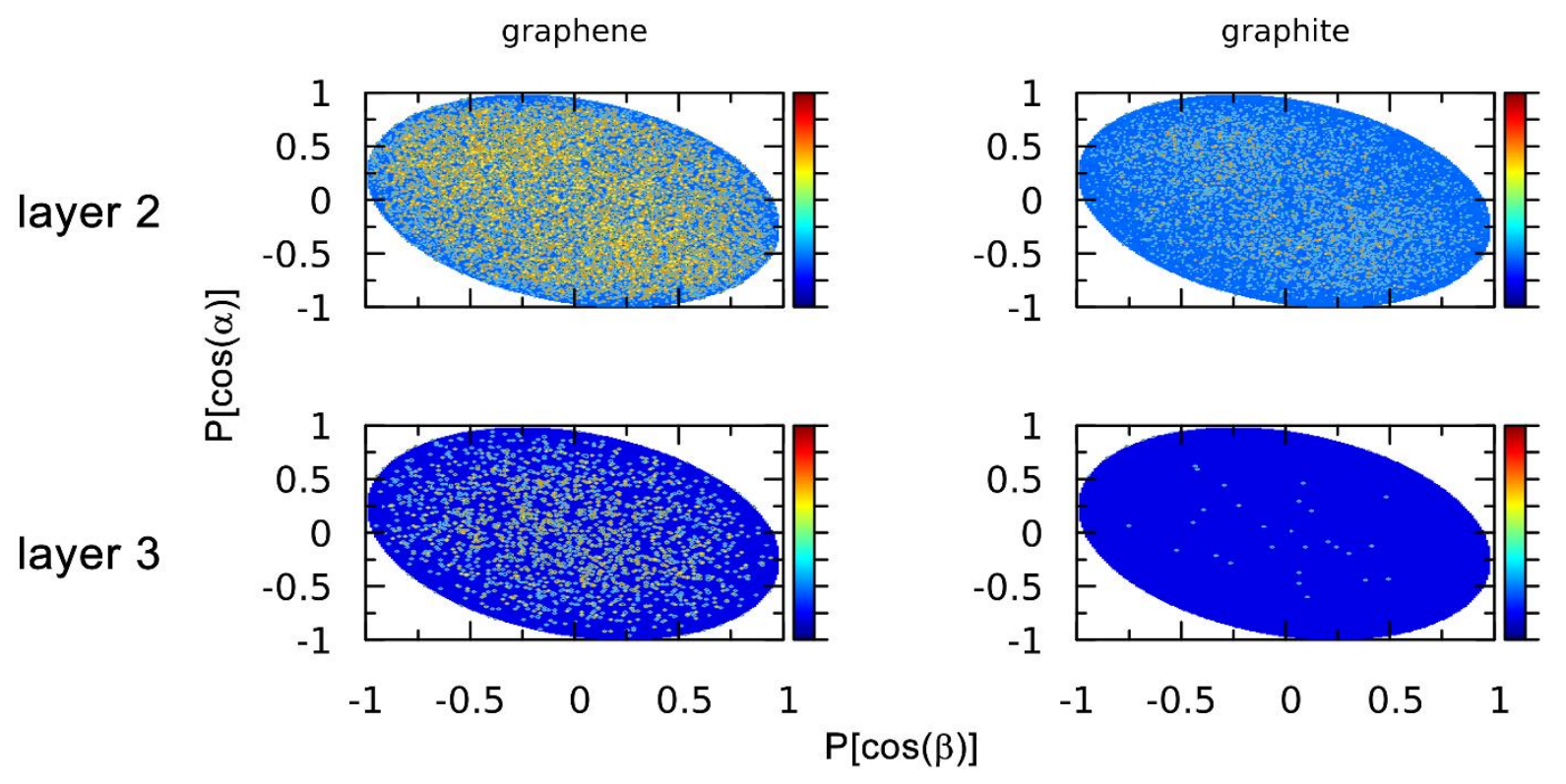

Figure S5 | Orientation of sub surface water molecules at graphitic interfaces. Joint probability distribution function of $\mathrm{OH}$ orientation angles for water molecules in layer 2 and layer 3, relative to the bulk. 


\section{REFERENCES}

1. Abascal, J. L.; Vega, C. A general purpose model for the condensed phases of water: TIP4P/2005. J. Chem. Phys. 2005, 123 (23), 234505.

2. Plimpton, S. FAST PARALLEL ALGORITHMS FOR SHORT-RANGE MOLECULAR-DYNAMICS. J. Comput. Phys. 1995, 117 (1), 1-19.

3. Pascal, T. A.; Karasawa, N.; Goddard III, W. A. Quantum mechanics based force field for carbon (QMFF-CX) validated to reproduce the mechanical and thermodynamics properties of graphite. J. Chem. Phys. 2010, 133 (13), 134114.

4. Werder, T.; Walther, J. H.; Jaffe, R.; Halicioglu, T.; Koumoutsakos, P. On the water-carbon interaction for use in molecular dynamics simulations of graphite and carbon nanotubes. J. Phys. Chem. B 2003, 107 (6), 1345-1352.

5. Yeh, I.-C.; Berkowitz, M. L. Ewald summation for systems with slab geometry. J. Chem. Phys. 1999, 111 (7), 3155-3162.

6. Hockney, R. W.; Eastwood, J. W. Computer simulation using particles. 1st ed.; Taylor \& Francis Group: Boca Raton, 1988; p 540.

7. Shinoda, W.; Shiga, M.; Mikami, M. Rapid estimation of elastic constants by molecular dynamics simulation under constant stress. Phys. Rev. B 2004, 69 (13).

8. Martyna, G. J.; Tobias, D. J.; Klein, M. L. Constant pressure molecular dynamics algorithms. J. Chem. Phys. 1994, 101 (5), 4177-4189.

9. Parrinello, M.; Rahman, A. Polymorphic transitions in single crystals: A new molecular dynamics method. J. Appl. Phys. 1981, 52 (12), 7182-7190.

10. Tuckerman, M. E.; Alejandre, J.; López-Rendón, R.; Jochim, A. L.; Martyna, G. J. A Liouvilleoperator derived measure-preserving integrator for molecular dynamics simulations in the isothermalisobaric ensemble. J. Phys. A: Math. Gen. 2006, 39 (19), 5629.

11. Lin, S. T.; Blanco, M.; Goddard, W. A. The two-phase model for calculating thermodynamic properties of liquids from molecular dynamics: Validation for the phase diagram of Lennard-Jones fluids. J. Chem. Phys. 2003, 119 (22), 11792-11805.

12. Lin, S.-T.; Maiti, P. K.; Goddard, W. A. Two-Phase Thermodynamic Model for Efficient and Accurate Absolute Entropy of Water from Molecular Dynamics Simulations. J. Phys. Chem. B 2010, 114 (24), 8191-8198.

13. Pascal, T. A.; Lin, S.-T.; Goddard III, W. A. Thermodynamics of liquids: standard molar entropies and heat capacities of common solvents from 2PT molecular dynamics. Phys. Chem. Chem. Phys. 2011, 13 (1), 169-181.

14. Pascal, T. A.; Goddard III, W. A. Hydrophobic Segregation, Phase Transitions and the Anomalous Thermodynamics of Water/Methanol Mixtures. J. Phys. Chem. B 2012, 116 (47), 13905-13912.

15. Pannir Sivajothi, S. S.; Lin, S.-T.; Maiti, P. K. Efficient computation of entropy and other thermodynamic properties for two-dimensional systems using two-phase thermodynamic model. J. Phys. Chem. B 2018, 123 (1), 180-193.

16. Allen, M. P.; Tildesley, D. J. Computer simulation of liquids. Clarendon Press ; Oxford University Press: Oxford [England]; New York, 1987.

17. Eyring, H.; Ree, T. SIGNIFICANT LIQUID STRUCTURES, VI. THE VACANCY THEORY OF LIQUIDS. Proc. Natl. Acad. Sci. U. S. A. 1961, 47 (4), 526-537.

18. Carnahan, N. F.; Starling, K. E. THERMODYNAMIC PROPERTIES OF A RIGID-SPHERE FLUID. J. Chem. Phys. 1970, 53 (2), 600-603.

19. McQuarrie, D. A. Statistical mechanics. University Science Books: Sausalito (Calif.), 2000. 
20. Desjarlais, M. P. First-principles calculation of entropy for liquid metals. Phys. Rev. E 2013, 88 (6), 062145.

21. Berendsen, H. J. C.; Grigera, J. R.; Straatsma, T. P. The missing term in effective pair potentials. J. Phys. Chem. 1987, 91 (24), 6269-6271.

22. Jorgensen, W. L.; Chandrasekhar, J.; Madura, J. D.; Impey, R. W.; Klein, M. L. Comparison of simple potential functions for simulating liquid water. J. Chem. Phys. 1983, 79 (2), 926-935.

23. Misra, R. P.; Blankschtein, D. Insights on the role of many-body polarization effects in the wetting of graphitic surfaces by water. J. Phys. Chem. C 2017, 121 (50), 28166-28179.

24. Pascal, T. A.; Goddard, W. A.; Jung, Y. Entropy and the driving force for the filling of carbon nanotubes with water. Proc. Natl. Acad. Sci. U. S. A. 2011, 108 (29), 11794-11798.

25. Scocchi, G.; Sergi, D.; D'Angelo, C.; Ortona, A. Wetting and contact-line effects for spherical and cylindrical droplets on graphene layers: A comparative molecular-dynamics investigation. Phys. Rev. $E$ 2011, 84 (6), 061602.

26. Luzar, A.; Chandler, D. Hydrogen-bond kinetics in liquid water. Nature 1996, 379 (6560), 55-57. 27. González, M. A.; Abascal, J. L. F. A flexible model for water based on TIP4P/2005. J. Chem. Phys. 2011, 135 (22), 224516. 